\title{
Regulation of the actin cytoskeleton in Helicobacter pylori-induced migration and invasive growth of gastric epithelial cells
}

\author{
Silja Wessler ${ }^{1 *}$, Mario Gimona² and Gabriele Rieder ${ }^{1}$
}

\begin{abstract}
Dynamic rearrangement of the actin cytoskeleton is a significant hallmark of Helicobacter pylori (H. pylori) infected gastric epithelial cells leading to cell migration and invasive growth. Considering the cellular mechanisms, the type IV secretion system (T4SS) and the effector protein cytotoxin-associated gene A (CagA) of H. pylori are well-studied initiators of distinct signal transduction pathways in host cells targeting kinases, adaptor proteins, GTPases, actin binding and other proteins involved in the regulation of the actin lattice. In this review, we summarize recent findings of how $\mathrm{H}$. pylori functionally interacts with the complex signaling network that controls the actin cytoskeleton of motile and invasive gastric epithelial cells.
\end{abstract}

Keywords: Helicobacter pylori, type IV secretion system, CagA, actin cytoskeleton

\section{Review}

The continuous reorganization and turnover of the actin cytoskeleton is a fundamental process in the regulation of cell adhesion to neighboring cells and extracellular matrix (ECM), phagocytosis, cell shape or migration. Generally, actin exists in cells as monomeric globular actin (G-actin) and filamentous actin (F-actin), which are formed upon polymerization of G-actin monomers in a defined directionality. A wide range of upstream signaling molecules including the cell adhesion molecule E-cadherin, integrins, components of the ECM, or stimuli such as tumor necrosis factor alpha (TNF- $\alpha$ ) and lysophosphatidic acid (LPA) are known in the transmission of extracellular signals to the actin cytoskeleton allowing rapid reactions to a changing environment (Figure 1A). Hence, remodeling of the actin cytoskeleton architecture depends on a large group of signaling molecules that bind to actin and modulate the assembly of the actin network (see [1] for a comprehensive overview).

Among actin-dependent cellular processes, efficient cell migration requires a coordinated rearrangement of the actin lattice in motile cells. Polymerization of F-actin at

\footnotetext{
* Correspondence: silja.wessler@sbg.ac.at

'Division of Molecular Biology, Department of Microbiology, University of Salzburg, Salzburg, Austria

Full list of author information is available at the end of the article
}

cell protrusions triggers the formation of sheet-like lamellipodia and rod-like filopodia pushing migrating cells $[2,3]$. Additionally, formation of contractile structures through interaction of actin with myosin II pulls the cell body across the ECM. Those processes involve a wide range of actin binding proteins (e.g. cortactin, $\alpha$ actinin, fascin, profilin, filamin, etc.) that contribute to actin stabilization, bundling and branching, forming a complex network. Signaling pathways modulating actin rearrangement are complex and have been covered in several excellent reviews [4-6]. Summarizing the most important findings in a simplistic model (Figure 1A), signaling pathways initiated at cell surface receptors to promote distinct membrane protrusions converge on Rho family GTPases as the key elements of signal transduction. One of the best characterized Rho GTPase family members is RhoA regulating the formation of stress fibers and focal adhesion assembly, while Rac and Cdc42 are mainly involved in membrane ruffling and formation of filopodia, respectively [4]. Rac1 and Cdc42 can induce actin polymerization through members of the WiskottAldrich syndrome protein (WASP) family and WASPinteracting proteins (WIPs). The WASP family of actin nucleation promoting factors (NPFs) includes WASP, NWASP and four forms of WASP verprolin homologous protein (WAVE). Through a conserved C-terminal 


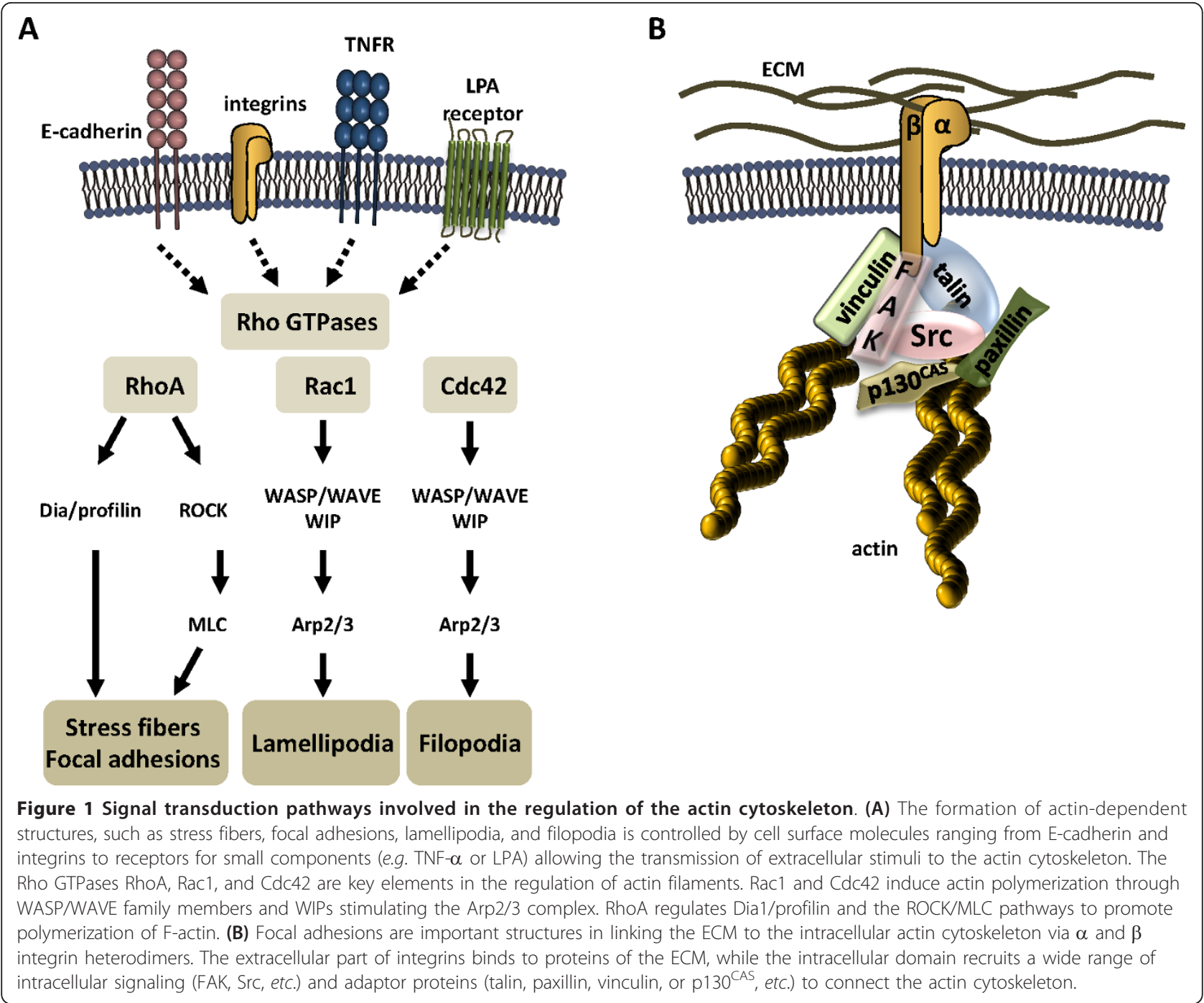

domain, WASP proteins stimulate the actin-related proteins $2 / 3$ (Arp2/3) complex activity to nucleate actin filaments and to elongate at their free barbed ends. Stress fiber assembly and contraction are predominantly induced by RhoA [7] as mentioned above, which controls Dia1/profilin to promote polymerization of F-actin [8]. Another mechanism involves Rho-induced Rho-associated serine/threonine kinase (ROCK) as an important downstream effector to induce myosin light chain (MLC) phosphorylation [9] (Figure 1A).

Commonly, contractile stress fibers attach to the plasma membrane at nascent focal adhesions, which are stabilized by $\alpha$ and $\beta$ integrin heterodimeric receptors (Figure 1B). Bridging the ECM to the actin cytoskeleton, the integrin ectodomain directly binds to ECM proteins (e.g. fibronectin), while the intracellular domain is connected to the actin cytoskeleton via recruited adaptor and signaling proteins including focal adhesion kinase (FAK), vinculin, talin and paxillin [6]. Upon activation, FAK recruits the nonreceptor tyrosine kinase $\mathrm{c}$-Src to the focal adhesion sites in order to phosphorylate other focal adhesion proteins such as paxillin and $\mathrm{p} 130^{\mathrm{Cas}}$ leading to mature focal adhesions (Figure 1B). The integrity and maturation of focal adhesion complexes cycle between assembly at the protrusions and disassembly at the trailing edge of migrating cells; however the precise molecular mechanisms are not completely understood. In this review, we summarize the current findings on how the human carcinogen Helicobacter pylori (H. pylori) controls the host cell actin cytoskeleton to form stress fibers and deregulates adhesion complexes to induce changes in cell shape, migration and invasive growth.

\section{H. pylori induces migration and invasive growth of gastric epithelial cells}

H. pylori is one of the most successful human pathogen that colonizes the gastric lining epithelium in the 
stomach of approximately $50 \%$ of the world's population. Once acquired and not eradicated by antibiotics, H. pylori normally persists throughout lifespan since the host is unable to clear the infection. Only a minority of $10-15 \%$ of infected individuals develops severe gastric diseases which mainly depend on bacterial expressed pathogenic and virulence factors, environmental determinants and individual genetic predispositions (e.g. polymorphisms of host genes such as interleukin- $1 \beta$ (IL-1 $\beta$ ), IL-8, IL-10, runt-related gene 3 (RUNX3), etc.), which can influence gastric atrophy and carcinogenesis [10-12]. Most severe complications are inflammatory disorders involving acute and chronic gastritis or ulceration of the stomach and duodenum, which can eventually result in Mucosa Associated Lymphoid Tissue (MALT) lymphoma and gastric cancer [13]. According to its capability to promote cancer, $H$. pylori was classified by the World Health Organization as a class-I carcinogen [14].

H. pylori pathogenesis is dependent on the expression of bacterial virulence factors [10], which might involve complex cellular responses of gastric epithelial cells $[15,16]$. The vacuolating cytotoxin A (VacA) is secreted by many, if not all, $H$. pylori isolates and might enhance the $H$. pylori virulence though its pleiotropic functions in vivo. VacA binds to many surface factors, including the receptor-like protein tyrosine phosphatase alpha and beta (RPTP $\alpha$ and RPTP $\beta$ ) presented on host cells and, after uptake, induces membrane anion-selective channels and pore formation, apoptosis and gigantic vacuoles in host cells [17]. VacA is further associated with the inhibition of $\mathrm{T}$-cell function through binding to the $\beta 2$ integrin receptor $[18,19]$. Another important pathogenic factor is the cytotoxin-associated gene A ( CagA), which has attracted much attention since its expression is closely associated with the development of severe diseases in vivo [20,21]. The cagA gene is located within the cag pathogenicity island (cagPAI) region on the bacterial chromosome, which encodes proteins important for structure and function of a specialized type IV secretion system (T4SS) $[22,23]$. Importantly, it has been demonstrated that the cagPAI protein CagL represents a T4SSpilus associated adhesin for $\alpha 5 \beta 1$ integrin expressed on the epithelial host cell surface. Binding of the fibronectinmimicking Arg-Gly-Asp (RGD) motif in the CagL molecule to $\beta 1$ integrin is necessary to translocate CagA into the host cytoplasm $[24,25]$. Many studies described that CagA-positive H. pylori strains are closely connected with the development of acute gastritis, pre-neoplastic and neoplastic lesion [26-29]. Causative associations between CagA and the formation of neoplasia were demonstrated in Mongolian gerbils [30,31] and in a transgenic mouse model in which CagA induced neoplastic transformations in vivo [32].
In healthy individuals, the gastric epithelium represents effective first barriers against pathogens, which is tightly sealed by coordinated regulation of epithelial cell shape, polarity, cell-to-cell and cell-to-matrix adhesions. Concomitantly with colonization of the gastric mucus, $H$. pylori dismantles the epithelial barrier function to induce inflammatory responses and neoplastic changes dependent on $H$. pylori virulence factors [33]. This might be facilitated by a rearrangement of the actin cytoskeleton as a central mechanism in those processes. Supporting this suggestion, H. pylori induces the formation of protrusions and massive stress fibers in cultured gastric epithelial cells accompanied by the loss of epithelial morphology and cell-to-cell adhesions leading to a mitogenic-invasive scattering phenotype in vitro $[33,34]$ reminiscent of growth factor-induced Epithelial-Mesenchymal Transition (EMT). The EMT phenotype requires a complex morphogenetic program initiated by alteration of gene expression, the loss of typical epithelial properties and the increase of mesenchymal characteristics [35], which could be detected in $H$. pylori-colonized cells [36]. During EMT, cells lose their polar, epithelial nature and acquire a highly motile, mesenchymal morphology. Principally, EMT is defined by the (i) disassembly of intercellular junctions, (ii) reorganization of the actin cytoskeleton from cell-cell and cell-matrix junctions into protrusive and invasive pseudopodial structures such as actin stress fibers and actin-dependent protrusion of cell pseudopodia, (iii) and an increase of cell motility. In general, these processes occur in synchronous fashion, but independently from each other [35]. Accordingly, efficient $H$. pylori-mediated cell migration is an extremely complex coordinated process which is initiated by the extension of lamellipodia at the leading edge of the cell, assembly of new focal adhesion complexes, secretion of proteases to degrade contacts to the ECM supporting the formation of invadopodia, development of contractile forces and finally disassembly of focal adhesions leading to tail detachment (Figure 2) [34,37].

Actin-dependent protrusion of pseudopodial surface extensions is a key element during EMT-related migration of $H$. pylori-colonized cells. Pathogenic H. pylori strains induce a morphogenetic program in different gastric epithelial cell lines that closely resembles the features of EMT [36]. CagA-transfected cells invade through the extracellular matrix via the formation of invasive pseudopodia [38] indicating that CagA might induce EMT in gastric cancer cells. Functionally these structures mimic invasive podosomes or invadopodia, and show a similar dependence on matrix metalloproteases (MMPs) for invasion. In support of this concept non-invasive podosomes have been shown to become gradually replaced by invasive invadopodia in EMT (Figure 2) [39]. 


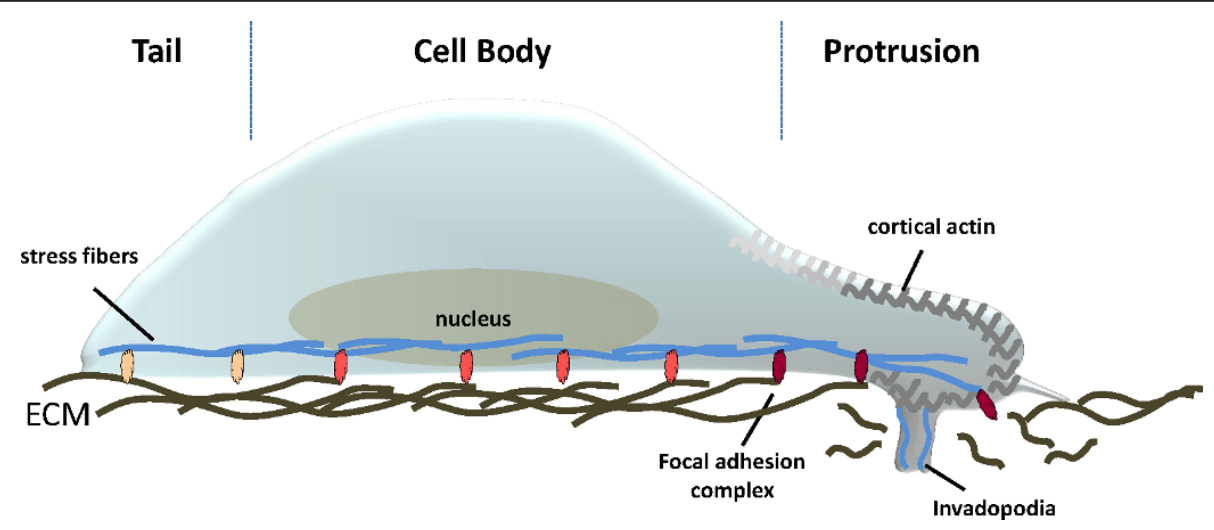

Figure 2 Model of migrating epithelial cells. For efficient migration, epithelial cells develop new actin-dependent protrusions which are connected to the ECM via newly assembled focal adhesions (red) at the leading edge. Secretion of proteases to degrade ECM is required to extend the protrusion into the ECM to form invadopodia. At the tail, matured focal adhesions (grey) disassemble to facilitate the movement of the cell body in a defined direction.

Adhesion based spatio-temporal orchestration of actinpolymerization-driven invasive structures $[40]$ is a feature of many physiological and pathological events. The players in this scenario are mechano-sensitive molecules that also depend on integrin-mediated outside-in signaling cascades and involve many of the same players (such as ezrin, Abl, Src, etc.) that are required for $H$. pylori-induced cell invasion into neighboring tissues $[38,41,42]$. A significant element that separates invadopodia from focal adhesions is the modulation of the cell's secretory machinery and the focal secretion of ECM-degrading matrix metalloproteases (MMPs) that ultimately allow the breach of tissue boundaries [43]. The ultrastructural features and intracellular dynamics of $H$. pylori-induced pseudopods are still poorly defined, but a future identification of these structures as invadopodia-related cellular protrusions would not be a surprise.

As infection with cagA-positive strains of $H$. pylori is tightly associated with the induction of gastric adenocarcinoma the targets highjacked by injected CagA likely controls pseudopod formation and invasion of infected motile cells. Indeed, in vitro studies have shown that CagA binds the adapter molecule growth factor receptor bound protein 2 (Grb2) [44], which can link Abl and Src kinase signaling cascades to MMP expression and invadopodium formation [45] and can thus contribute to the site-specific formation of signaling complexes required for cell migration and invasive growth. Interestingly, $H$. pylori induces expression of MMP-7 at the lamellipodia of motile cells, which was also triggered by activated RhoA and Rac [46], suggesting a close connection between ECM degradation, invasive growth and efficient cell motility. The cortical cytoskeleton serves as a nexus between the extracellular environment and the cytoplasm, and is positioned to coordinate cellular signal relays. It comes thus as no surprise that cytoskeleton-associated cortical proteins have key roles in H. pylori-induced cell modulation. The mucin-like transmembrane glycoprotein podoplanin can also induce EMT, cell migration and invasive growth by recruiting the ERM (Ezrin, Radixin, Moesin)-family protein ezrin, an organizer of the cortical cytoskeleton, to the plasma membrane. This interaction is essential for the activation of the RhoA/ROCK pathway by podoplanin [47,48]. In H. pyloriinfected gastric epithelial cells, ezrin becomes dephosphorylated which could be involved in the development and metastasis of $H$. pylori-induced gastric cancer [49]. Ezrin's dual role as an actin binding and GTPase scaffolding protein further identifies this molecular complex as a key target for understanding the cytoskeletal rearrangements that lead to migration and invasive growth of infected epithelial cells $[49,50]$.

In fact, the EMT-like phenotype of H. pylori-infected epithelial host cells implies the formation of protrusions and elongation. Rather unfortunate, terms like 'scattering phenotype' or 'hummingbird phenotype' in connection with $H$. pylori infection has been widely become synonymous with 'cell elongation' or 'cell migration'. Interestingly, data are accumulating indicating that cellular elongation and motility are differentially regulated by H. pylori via independent signal transduction pathways [51]. Consistently observed, the drastic elongation of host cells is strictly dependent on CagA injection [52-54], while H. pylori-induced cell motility is cagPAI-dependent, but largely CagA-independent $[42,51,55]$. Making those observations more complex, data are accumulating that CagA and VacA functions are antagonizing each other in some assays. In accordance with a study showing that specific VacA variants inhibited CagA-dependent cell elongation, CagA reduced VacA-mediated apoptosis and vice versa, underscoring the interfering functions of pathogenic factors expressed by $H$. pylori [56,57]. Furthermore, H. pylori-expressed pathogenic factors might differentially 
interact with host cells leading to the disruption of the gastric epithelium and determining the outcome of gastric disorders. Rapid host cell elongation and migration are particular evident in human gastric cancer cells (e.g. AGS cells) $[53,58,59]$, breast cancer cells (e.g. MCF-7 cells) $[42,60]$, and a subtype of the canine kidney cell line MDCK [55,61]. Milder and less pronounced development of this typical phenotype was observed in gastric MKN-1, MKN-28 and Hs-746T cells within early phases of H. pylori infections [15]. In terms of cell morphological and junctional changes, only few reports on primary gastric epithelial cells are available $[62,63]$. Importantly, Krüger et al. demonstrated $H$. pylori-induced motility and growth of ex vivo isolated gastric cells [63]. Access to primary cells is limited; hence it is important to investigate observed cellular molecular mechanisms in vivo as well. So far, it is still speculative if changes in cell morphology actually contribute to $H$. pylori-associated gastric diseases, even these processes likely influence host responses during decades of persistent $H$. pylori infections.

\section{Helicobacter pylori induced signal transduction pathways leading to a deregulated actin cytoskeleton independently of CagA}

While it is clear that H. pylori induces striking cytoskeletal changes in epithelial cells, knowledge of the signal transduction pathways is rare. In serum-starved cells, both CagA-positive and CagA-negative H. pylori strains mediated the formation of actin filaments and lamellipodial structures [64] implying activation of Rho GTPases. In fact, activation of Rac1 and Cdc42 has been demonstrated in H. pylori-infected AGS cells [65]. Microinjection of inactive Rac prevented actin cytoskeleton rearrangements in lamellipodial structures in H. pylori-colonized cells [64]. Through transfection of dominant-negative and catalyticactive cDNA constructs or using well-characterized GTPase-targeting toxins, Crk adapter proteins, Rac1 and $\mathrm{H}$-Ras, but not RhoA or Cdc42 were identified as crucial components leading to $H$. pylori-induced cell elongation [66]. Consistent with actin polymerization in H. pyloriinfected cells [64], activation of Rho GTPases occurs independently of CagA injection, but obviously required the T4SS apparatus [65]. Since CagL was identified as an adhesin for $\alpha 5 \beta 1$ integrins that is decorated at the tip of the T4SS allowing CagA injection and $\beta 1$ integrin activation [24], it is tempting to speculate that CagL represents a promising candidate for stimulating Rho GTPase activation as well (Figure 3A). This hypothesis is currently supported by the finding that CagL-coated latex beads stimulated membrane ruffling via integrin-mediated activation of FAK and Src [24]. Another possible scenario proposes is OipA as an inducing factor since oipA mutants have been reported to less activate FAK presumably inde- pendently of cagPAI or CagA [67]; however experiments with recomplemented oipA mutants are pending.

The $\beta 1$ integrin/FAK/Src pathway transmits signals to the actin cytoskeleton via paxillin, an important scaffolding protein located in focal adhesions [34]. In H. pyloriinfected cells activated FAK phosphorylates tyrosine 118 in the paxillin protein (paxillin ${ }^{\mathrm{Y} 118}$ ) which was essential for cell motility in response to H. pylori [68]. Since phosphorylated paxillin ${ }^{\mathrm{Y} 118}$ binds the adaptor protein $\mathrm{v}$-crk sarcoma virus CT10 oncogene homolog (Crk) in response to cell adhesion, platelet-derived growth factor (PDGF) or angiotensin II [69], H. pylori-triggered paxillin ${ }^{\mathrm{Y} 118}$ phosphorylation may also act upstream of the activation of Crk/DOCK180 (dedicator of cytokinesis)/Rac1/WAVE/ Arp2/3 signal transduction pathway in $H$. pylori-infected cells, which has been detected in another study (Figure 3A) [70]. Alternatively, H. pylori-induced Src activity could activate $\mathrm{p} 130^{\mathrm{Cas}}$ leading to the recruitment of the Crk complex; however an involvement of $\mathrm{p} 130^{\mathrm{Cas}}$ in H. pylori-mediated cytoskeletal rearrangement still needs to be demonstrated (Figure 3A).

\section{Regulation of CagA-mediated host cell elongation}

The $H$. pylori-induced changes in cell morphology are dominated by the drastic elongation of epithelial cells which involves active regulation of both the actin cytoskeleton and focal adhesions. Single-cell analyses suggested that $H$. pylori-dependent cell elongation might be mediated by deregulated focal adhesions rather than actin cytoskeleton rearrangement. Stabilized focal adhesions cause a defect in cell retraction leading to the formation of strong traction forces on motile $H$. pylori-infected cells [52]. CagA increases phosphorylation and subsequent activation of myosin light chain (MLC) in a Drosophila model [71]. The concomitant mispatterning of MLC results in cell elongation due to retraction failure and disruption of epithelial morphology and integrity. Based on a phosphoproteomic analysis the actin-binding protein vasodilatorstimulated phosphoprotein (VASP) was identified, which co-localized with focal adhesions of $H$. pylori-infected cells [72]. Down-regulation of VASP expression and inhibition of VASP phosphorylation blocked cell elongation in response to $H$. pylori, but it was not investigated whether phosphorylated VASP disturbed the disassembly of focal adhesions [72].

The significance of focal adhesions in promoting cell elongation has been emphasized by the finding that $\beta 1$ integrin-mediated injection of CagA is important in the process of cell elongation [24]. Upon translocation, CagA localizes at the inner membrane of infected cells, where it is rapidly phosphorylated by the non-receptor tyrosine kinases c-Src, Fyn, Lyn and Yes of the Src family kinases (SFK) $[73,74]$. Phosphorylation sites were localized in a 


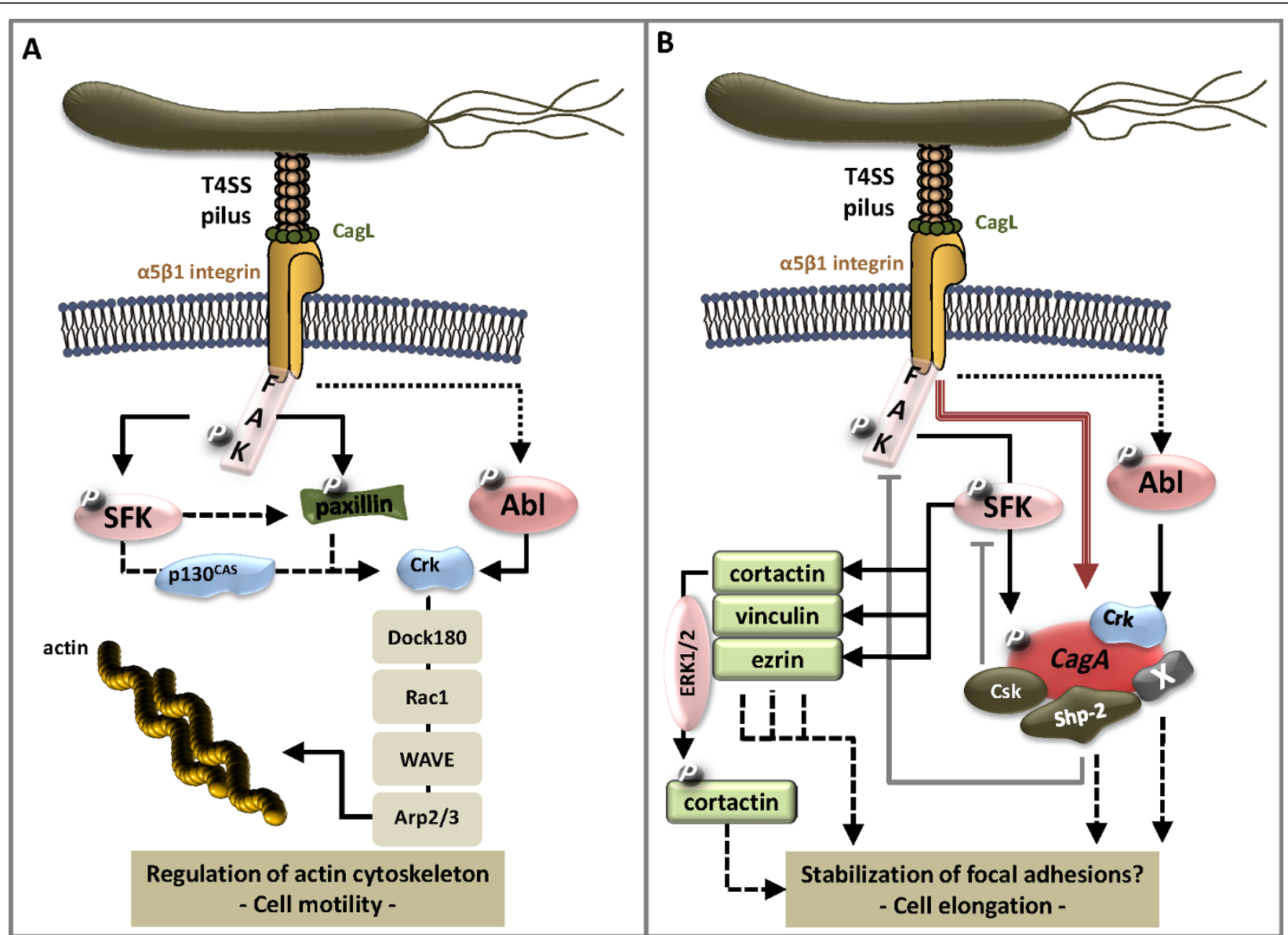

Figure 3 Schematic overview of CagL and CagA-mediated signal transduction pathways involved in $\mathrm{H}$. pylori-induced cell motility and elongation. (A) H. pylori expresses CagL at the tip of the T4SS that directly binds to $\beta 1$ integrins presented on gastric epithelial cells. Activated $\beta 1$ integrin stimulates FAK and Src activity in early phases of H. pylori infections. FAK phosphorylates paxillin upon infection which might contribute to c-Abl-phosphorylated Crk signaling, which could be influenced by SFK activity via paxillin or p130 CAS. FAK, SFKs and Abl kinasemediated activation of Crk proteins can regulate the actin cytoskeleton through the DOCK180/Rac1/WAVE/Arp2/3 pathway contributing to epithelial cell migration. (B) CagL-integrin binding leads to the translocation of the H. pylori pathogenic factor CagA into the host cytoplasm. CagA is rapidly phosphorylated by kinases of the Src family (SFK) and bind to a large number of host cells factors (X) in its phosphorylated and non-phosphorylated form. Tyrosine phosphorylated CagA interacts with Shp-2 and Csk to inactivate FAK and Src in late phases of H. pylori infection. While inactivated Src is replaced by activated Abl kinases to maintain CagA phosphorylation, inactive Src leads to tyrosine dephosphorylation of Src target molecules ezrin, vinculin and cortactin. Cortactin is then serine phosphorylated by H. pylori-activated ERK1/2 kinases, which crucially contributes to cell elongation. Black arrows, H. pylori-induced direct signaling pathways. Dotted arrows, H. pylori-induced or Src-mediated indirect signaling pathways. Grey arrows, inactivating signaling pathways. Red arrow, CagA injection as the central step in the regulation of focal adhesions. P, phosphorylated proteins. X, host cell proteins.

Glu-Pro-Ile-Tyr-Ala sequence (EPIYA motif), which exists as different 1-5 repeats, namely EPIYA-A, EPIYAB, EPIYA-C in Western H. pylori isolates and EPIYA-A, EPIYA-B, EPIYA-D in East-Asian strains [75,76]. The Src-mediated CagA phosphorylation $\left(\mathrm{CagA}^{\mathrm{PY}}\right)$ is followed by a rapid inactivation of Src kinase activity, triggered by the binding of CagA to the C-terminal Src kinase (Csk) (Figure 3B) [54,58]. Src kinase inactivation then leads to the dephosphorylation of Src target proteins such as vinculin, ezrin and cortactin $[49,54,77]$. In fact, tyrosine phosphorylation of $\mathrm{CagA}^{\mathrm{PY}}$ together with the dephosphorylation of SFKs and their target molecules are important in the process of regulation of the actin cytoskeleton and focal adhesions which contributes to the drastic morphological changes of $H$. pylori-infected cells (Figure 3B).

Another key molecule in $\mathrm{H}$. pylori-stimulated cell elongation is Shp-2 (src homology 2 domain tyrosine phosphatase) (Figure 3B). Analysis of ectopically expressed CagA and isogenic phosphorylation-resistant mutants revealed that $\mathrm{CagA}^{\mathrm{PY}}$ directly binds to Shp-2 which led to an increase of phosphatase activity of Shp-2 $[78,79]$. The CagA/Shp-2 complex has also been detected in the gastric mucosa of $H$. pylori-positive patients with gastritis 
and early stages of gastric cancer [80]. Activation of Shp-2 phosphatase activity has consequently been reported to inactivate FAK in cells that ectopically express CagA [81]. In contrast to activated FAK, dephosphorylated FAK cannot be localized in focal adhesions, which might support the development of the elongated cell phenotype. Contrary to this observation, CagL and OipA activate FAK in $H$. pylori-infected cells [24,67]. Recently, a new functional form of cortactin was reported, further underscoring the importance of cortactin as a critical mediator in signal transduction pathways in $H$. pyloriinfected host cells (Figure 3B). After Src-mediated tyrosine dephosphorylation, cortactin becomes phosphorylated at serine 405 (cortactin $^{\text {S405 }}$ ). Phosphorylated cortactin ${ }^{\text {S405 }}$ strongly binds to and activates FAK. Cortactin ${ }^{\mathrm{S} 405}$ phosphorylation was mediated by ERK1/2 kinases and might trap activated FAK leading to a disturbed turnover of focal adhesions (Figure 3B) [82]. This is one of the first identified mechanisms explaining why activation of mitogenicactivated protein (MAP) kinases via Rap1 GTPases [83] or protein kinases C (PKCs) [84] in response to $H$. pylori infections can contribute to cell elongation $[61,70,82]$.

In contrast to dephosphorylated SFK target molecules, phosphorylation of CagA ${ }^{\mathrm{PY}}$ is potently sustained by activated Abl kinases after inactivation of Src [60,85]. Abl kinases maintain $\mathrm{CagA}^{\mathrm{PY}}$ phosphorylation and $\mathrm{CagA}^{\mathrm{PY}}$ dependent downstream effects, which are still not fully understood. Interestingly, it was indicated that transfected East Asian-type CagA induced significantly stronger effects on rat cell growth than the Western CagA [86], which are obviously attributable to the different EPIYA motifs and their binding affinities to Shp-2 [75]. As it is not clear if Src and Abl kinases prefer different EPIYA motifs or exhibit similar phosphorylation affinities, further analyses are necessary to investigate the SFK and Abl kinase-mediated CagA phosphorylation.

Activated c-Abl consequently also phosphorylates Crk adapter proteins $[60,85]$, which has been reported to interact with CagA $^{\mathrm{PY}}[70]$ linking a large $\mathrm{CagA}^{\mathrm{PY}}$ recruited protein complex with signal transduction pathways towards the actin cytoskeleton (Figure 3B). Diverse, but coordinated signal transduction pathways converge on CagA ${ }^{\mathrm{PY}}$ as an important central key molecule in $H$. pylorimediated cell migration [76]. Beside Shp-2 as the first identified binding partner of CagA [78], many more binding partners for phosphorylated and non-phosphorylated CagA have been identified during the last years including Par1/MARK, c-Met, PLC $\gamma$ (Phospholipase C gamma), ZO1 (Zonula occludens-1), Csk (c-Src tyrosine kinase), Gab1 (Grb-associated binder 1), Crk (CDC2-related protein kinase) proteins, Grb2 and the cell adhesion protein Ecadherin [10,33]. It is still unclear whether one CagA molecule can bind to more than one interaction partner simultaneously. But for most of these identified binding proteins it could be shown that they play a role in the induction of the $H$. pylori-dependent scatter phenotype.

\section{Conclusions}

Infection of gastric epithelial cells with $H$. pylori in vitro induces a strong motility response; however, our current understanding of the complex molecular mechanism contributing to this phenotype is still rudimentary understood. Although data are steadily increasing indicating that $\alpha 5 \beta 1$ integrin/CagA signaling is involved in stabilization of focal adhesion at the rear of the motile cell, it is unclear how these processes can be differentiated from the cellular mechanisms stimulating the assembly of nascent focal adhesions and rearrangement of the actin cytoskeleton at the leading edge. Hence, further studies are necessary to investigate signal transduction pathways controlling these locally demarcated regions in $H$. pylori infected host cells in vitro as well as in vivo, which might have consequences on the physiological balance and integrity of the gastric epithelium in vivo.

\section{Acknowledgements}

We thank Steffen Backert for critical reading of the manuscript and apologize to all colleagues whose important work could not be cited here.

\section{Author details}

${ }^{1}$ Division of Molecular Biology, Department of Microbiology, University of Salzburg, Salzburg, Austria. ${ }^{2}$ University Clinic for Blood Group Serology and Transfusion Medicine, PMU, General Hospital Salzburg, SALK, Salzburg, Austria.

\section{Authors' contributions}

SW, MG and GR wrote the manuscript. All authors read and approved the final manuscript.

\section{Competing interests}

The authors declare that they have no competing interests.

Received: 4 July 2011 Accepted: 1 November 2011

Published: 1 November 2011

\section{References}

1. Le Clainche C, Carlier MF: Regulation of actin assembly associated with protrusion and adhesion in cell migration. Physiol Rev 2008, 88:489-513.

2. Small JV, Stradal T, Vignal E, Rottner K: The lamellipodium: where motility begins. Trends Cell Biol 2002, 12:112-120.

3. Faix J, Rottner K: The making of filopodia. Curr Opin Cell Biol 2006, 18:18-25.

4. Ridley AJ: Rho GTPases and actin dynamics in membrane protrusions and vesicle trafficking. Trends Cell Biol 2006, 16:522-529.

5. Parsons JT, Horwitz AR, Schwartz MA: Cell adhesion: integrating cytoskeletal dynamics and cellular tension. Nat Rev Mol Cell Biol 2010, 11:633-643.

6. Tomar A, Schlaepfer DD: Focal adhesion kinase: switching between GAPs and GEFs in the regulation of cell motility. Curr Opin Cell Biol 2009, 21:676-683.

7. Schmitz AA, Govek EE, Bottner B, Van Aelst L: Rho GTPases: signaling, migration, and invasion. Exp Cell Res 2000, 261:1-12.

8. Watanabe N, Madaule P, Reid T, Ishizaki T, Watanabe G, Kakizuka A, Saito Y, Nakao K, Jockusch BM, Narumiya S: p140mDia, a mammalian homolog of Drosophila diaphanous, is a target protein for Rho small GTPase and is a ligand for profilin. EMBO J 1997, 16:3044-3056. 
9. Totsukawa G, Yamakita Y, Yamashiro S, Hartshorne DJ, Sasaki Y, Matsumura F: Distinct roles of ROCK (Rho-kinase) and MLCK in spatial regulation of MLC phosphorylation for assembly of stress fibers and focal adhesions in 3T3 fibroblasts. J Cell Biol 2000, 150:797-806.

10. Wroblewski LE, Peek RM Jr, Wilson KT: Helicobacter pylori and gastric cancer: factors that modulate disease risk. Clin Microbiol Rev 2010, 23:713-739.

11. Hishida A, Matsuo K, Goto Y, Hamajima N: Genetic predisposition to Helicobacter pylori-induced gastric precancerous conditions. World J Gastrointest Oncol 2010, 2:369-379.

12. Suzuki M, Suzuki H, Minegishi Y, Ito K, Nishizawa T, Hibi T: H. pyloriEradication Therapy Increases RUNX3 Expression in the Glandular Epithelial Cells in Enlarged-Fold Gastritis. J Clin Biochem Nutr 2010, 46:259-264.

13. Parsonnet J, Friedman GD, Vandersteen DP, Chang Y, Vogelman JH, Orentreich N, Sibley RK: Helicobacter pylori infection and the risk of gastric carcinoma. N Engl J Med 1991, 325:1127-1131.

14. Schistosomes, liver flukes and Helicobacter pylori. IARC Working Group on the Evaluation of Carcinogenic Risks to Humans. Lyon, 7-14 June 1994. IARC Monogr Eval Carcinog Risks Hum 1994, 61:1-241.

15. Schneider S, Carra G, Sahin U, Hoy B, Rieder G, Wessler S: Complex Cellular Responses of Helicobacter pylori-Colonized Gastric Adenocarcinoma Cells. Infect Immun 2011, 79:2362-2371.

16. Bauer B, Moese S, Bartfeld S, Meyer TF, Selbach M: Analysis of cell typespecific responses mediated by the type IV secretion system of Helicobacter pylori. Infect Immun 2005, 73:4643-4652.

17. Costa AC, Figueiredo C, Touati E: Pathogenesis of Helicobacter pylori infection. Helicobacter 2009, 14(Suppl 1):15-20

18. Gebert B, Fischer W, Weiss E, Hoffmann R, Haas R: Helicobacter pylori vacuolating cytotoxin inhibits T lymphocyte activation. Science 2003, 301:1099-1102.

19. Sewald X, Gebert-Vogl B, Prassl S, Barwig I, Weiss E, Fabbri M, Osicka R, Schiemann M, Busch DH, Semmrich M, Holzmann B, Sebo P, Haas R: Integrin subunit CD18 Is the T-lymphocyte receptor for the Helicobacter pylori vacuolating cytotoxin. Cell Host Microbe 2008, 3:20-29.

20. Basso D, Zambon CF, Letley DP, Stranges A, Marchet A, Rhead JL, Schiavon S, Guariso G, Ceroti M, Nitti D, Rugge M, Plebani M, Atherton JC: Clinical relevance of Helicobacter pylori cagA and vacA gene polymorphisms. Gastroenterology 2008, 135:91-99.

21. Palli D, Masala G, Del Giudice G, Plebani M, Basso D, Berti D, Numans ME, Ceroti M, Peeters PH, Bueno de Mesquita HB, Buchner FL, ClavelChapelon F, Boutron-Ruault MC, Krogh V, Saieva C, Vineis P, Panico S, Tumino R, Nyrén O, Simán H, Berglund G, Hallmans G, Sanchez MJ, Larrãnaga N, Barricarte A, Navarro C, Quiros JR, Key T, Allen N, Bingham S, et al: CagA+ Helicobacter pylori infection and gastric cancer risk in the EPIC-EURGAST study. Int J Cancer 2007, 120:859-867.

22. Fischer W: Assembly and molecular mode of action of the Helicobacter pylori Cag type IV secretion apparatus. FEBS J 2011, 278:1203-1212.

23. Backert S, Tegtmeyer N, Selbach M: The versatility of Helicobacter pylori CagA effector protein functions: The master key hypothesis. Helicobacter 2010, 15:163-176

24. Kwok T, Zabler D, Urman S, Rohde M, Hartig R, Wessler S, Misselwitz R, Berger J, Sewald N, Konig W, Backert S: Helicobacter exploits integrin for type IV secretion and kinase activation. Nature 2007, 449:862-866.

25. Tegtmeyer N, Hartig R, Delahay RM, Rohde M, Brandt S, Conradi J, Takahashi S, Smolka AJ, Sewald N, Backert S: A small fibronectin-mimicking protein from bacteria induces cell spreading and focal adhesion formation. J Biol Chem 2010, 285:23515-23526.

26. Smith MG, Hold GL, Tahara E, El-Omar EM: Cellular and molecular aspects of gastric cancer. World J Gastroenterol 2006, 12:2979-2990

27. Blaser MJ, Perez-Perez Gl, Kleanthous H, Cover TL, Peek RM, Chyou PH, Stemmermann GN, Nomura A: Infection with Helicobacter pylori strains possessing cagA is associated with an increased risk of developing adenocarcinoma of the stomach. Cancer Res 1995, 55:2111-2115.

28. Parsonnet J, Friedman GD, Orentreich N, Vogelman H: Risk for gastric cancer in people with CagA positive or CagA negative Helicobacter pylori infection. Gut 1997, 40:297-301.

29. Hatakeyama M: Helicobacter pylori and gastric carcinogenesis. Gastroenterol 2009, 44:239-248.
30. Rieder G, Merchant JL, Haas R: Helicobacter pylori cag-type IV secretion system facilitates corpus colonization to induce precancerous conditions in Mongolian gerbils. Gastroenterology 2005, 128:1229-1242.

31. Wiedemann T, Loell E, Mueller S, Stoeckelhuber M, Stolte M, Haas R, Rieder G: Helicobacter pylori cag-Pathogenicity island-dependent early immunological response triggers later precancerous gastric changes in Mongolian gerbils. PLoS One 2009, 4:e4754.

32. Ohnishi N, Yuasa H, Tanaka S, Sawa H, Miura M, Matsui A, Higashi H, Musashi M, Iwabuchi K, Suzuki M, Yamada G, Azuma T, Hatakeyama M: Transgenic expression of Helicobacter pylori CagA induces gastrointestinal and hematopoietic neoplasms in mouse. Proc Natl Acad Sci USA 2008, 105:1003-1008.

33. Wessler S, Backert S: Molecular mechanisms of epithelial-barrier disruption by Helicobacter pylori. Trends Microbiol 2008, 16:397-405.

34. Schneider S, Weydig C, Wessler S: Targeting focal adhesions:Helicobacter pylori-host communication in cell migration. Cell Commun Signal 2008, $6: 2$

35. Boyer B, Valles AM, Edme N: Induction and regulation of epithelialmesenchymal transitions. Biochem Pharmacol 2000, 60:1091-1099.

36. Yin Y, Grabowska AM, Clarke PA, Whelband E, Robinson K, Argent RH, Tobias A, Kumari R, Atherton JC, Watson SA: Helicobacter pylori potentiates epithelial:mesenchymal transition in gastric cancer: links to soluble HB-EGF, gastrin and matrix metalloproteinase-7. Gut 2010, 59:1037-1045.

37. Parri M, Chiarugi P: Rac and Rho GTPases in cancer cell motility control. Cell Commun Signal 2010, 8:23.

38. Bagnoli F, Buti L, Tompkins L, Covacci A, Amieva MR: Helicobacter pylori CagA induces a transition from polarized to invasive phenotypes in MDCK cells. Proc Natl Acad Sci USA 2005, 102:16339-16344.

39. Takkunen M, Hukkanen M, Liljestrom M, Grenman R, Virtanen I: Podosomelike structures of non-invasive carcinoma cells are replaced in epithelialmesenchymal transition by actin comet-embedded invadopodia. J Cell Mol Med 2010, 14:1569-1593.

40. Destaing $\mathrm{O}$, Block MR, Planus E, Albiges-Rizo C: Invadosome regulation by adhesion signaling. Curr Opin Cell Biol 2011

41. Oliveira MJ, Costa AC, Costa AM, Henriques L, Suriano G, Atherton JC, Machado JC, Carneiro F, Seruca R, Mareel M, Leroy A, Figueiredo C: Helicobacter pylori induces gastric epithelial cell invasion in a c-Met and type IV secretion system-dependent manner. J Biol Chem 2006, 281:34888-34896.

42. Weydig C, Starzinski-Powitz A, Carra G, Lower J, Wessler S: CagAindependent disruption of adherence junction complexes involves $\mathrm{E}$ cadherin shedding and implies multiple steps in Helicobacter pylori pathogenicity. Exp Cell Res 2007, 313:3459-3471.

43. Ayala I, Baldassarre M, Giacchetti G, Caldieri G, Tete S, Luini A, Buccione R: Multiple regulatory inputs converge on cortactin to control invadopodia biogenesis and extracellular matrix degradation. J Cell Sci 2008, 121:369-378.

44. Mimuro H, Suzuki T, Tanaka J, Asahi M, Haas R, Sasakawa C: Grb2 is a key mediator of helicobacter pylori CagA protein activities. Mol Cell 2002, 10:745-755.

45. Smith-Pearson PS, Greuber EK, Yogalingam G, Pendergast AM: Abl kinases are required for invadopodia formation and chemokine-induced invasion. J Biol Chem 2010, 285:40201-40211.

46. Wroblewski LE, Noble PJ, Pagliocca A, Pritchard DM, Hart CA, Campbell F, Dodson AR, Dockray GJ, Varro A: Stimulation of MMP-7 (matrilysin) by Helicobacter pylori in human gastric epithelial cells: role in epithelial cell migration. J Cell Sci 2003, 116:3017-3026.

47. Martin-Villar E, Megias D, Castel S, Yurrita MM, Vilaro S, Quintanilla M: Podoplanin binds ERM proteins to activate RhoA and promote epithelial-mesenchymal transition. J Cell Sci 2006, 119:4541-4553.

48. Fernandez-Munoz B, Yurrita MM, Martin-Villar E, Carrasco-Ramirez P, Megias D, Renart J, Quintanilla M: The transmembrane domain of podoplanin is required for its association with lipid rafts and the induction of epithelial-mesenchymal transition. Int J Biochem Cell Biol 2011, 43:886-896

49. Selbach M, Moese S, Backert S, Jungblut PR, Meyer TF: The Helicobacter pylori CagA protein induces tyrosine dephosphorylation of ezrin. Proteomics 2004, 4:2961-2968. 
50. Fan LL, Chen DF, Lan CH, Liu KY, Fang DC: Knockdown of ezrin via RNA interference suppresses Helicobacter pylori-enhanced invasion of gastric cancer cells. Cancer Biol Ther 2011, 11:746-752

51. Moese S, Selbach M, Kwok T, Brinkmann V, Konig W, Meyer TF, Backert S: Helicobacter pylori induces AGS cell motility and elongation via independent signaling pathways. Infect Immun 2004, 72:3646-3649.

52. Bourzac KM, Botham CM, Guillemin K: Helicobacter pylori CagA induce AGS cell elongation through a cell retraction defect that is independent of Cdc42, Rac1, and Arp2/3. Infect Immun 2007, 75:1203-1213.

53. Segal ED, Cha J, Lo J, Falkow S, Tompkins LS: Altered states: involvement of phosphorylated CagA in the induction of host cellular growth changes by Helicobacter pylori. Proc Natl Acad Sci USA 1999, 96:14559-14564.

54. Selbach M, Moese S, Hurwitz R, Hauck CR, Meyer TF, Backert S: The Helicobacter pylori CagA protein induces cortactin dephosphorylation and actin rearrangement by c-Src inactivation. EMBO J 2003, 22:515-528.

55. Al-Ghoul L, Wessler S, Hundertmark T, Kruger S, Fischer W, Wunder C, Haas R, Roessner A, Naumann M: Analysis of the type IV secretion systemdependent cell motility of Helicobacter pylori-infected epithelial cells. Biochem Biophys Res Commun 2004, 322:860-866.

56. Oldani A, Cormont M, Hofman V, Chiozzi V, Oregioni O, Canonici A, Sciullo A, Sommi P, Fabbri A, Ricci V, Boquet P: Helicobacter pylori counteracts the apoptotic action of its VacA toxin by injecting the CagA protein into gastric epithelial cells. PLoS Pathog 2009, 5:e1000603.

57. Tegtmeyer N, Zabler D, Schmidt D, Hartig R, Brandt S, Backert S: Importance of EGF receptor, HER2/Neu and Erk1/2 kinase signalling for host cell elongation and scattering induced by the Helicobacter pylori CagA protein: antagonistic effects of the vacuolating cytotoxin VacA. Cell Microbiol 2009, 11:488-505.

58. Tsutsumi R, Higashi H, Higuchi M, Okada M, Hatakeyama M: Attenuation of Helicobacter pylori CagA $\times$ SHP-2 signaling by interaction between CagA and C-terminal Src kinase. J Biol Chem 2003, 278:3664-3670.

59. Backert S, Moese S, Selbach M, Brinkmann V, Meyer TF: Phosphorylation of tyrosine 972 of the Helicobacter pylori CagA protein is essential for induction of a scattering phenotype in gastric epithelial cells. Mol Microbiol 2001, 42:631-644.

60. Poppe M, Feller SM, Romer G, Wessler S: Phosphorylation of Helicobacter pylori CagA by c-Abl leads to cell motility. Oncogene 2007, 26:3462-3472.

61. Churin Y, Al-Ghoul L, Kepp O, Meyer TF, Birchmeier W, Naumann M: Helicobacter pylori CagA protein targets the c-Met receptor and enhances the motogenic response. J Cell Biol 2003, 161:249-255.

62. Conlin VS, Curtis SB, Zhao Y, Moore ED, Smith VC, Meloche RM, Finlay BB, Buchan AM: Helicobacter pylori infection targets adherens junction regulatory proteins and results in increased rates of migration in human gastric epithelial cells. Infect Immun 2004, 72:5181-5192

63. Krueger $\mathrm{S}$, Hundertmark T, Kuester D, Kalinski T, Peitz U, Roessner A: Helicobacter pylori alters the distribution of ZO-1 and p120ctn in primary human gastric epithelial cells. Pathol Res Pract 2007, 203:433-444

64. Palovuori R, Perttu A, Yan Y, Karttunen R, Eskelinen S, Karttunen TJ: Helicobacter pylori induces formation of stress fibers and membrane ruffles in AGS cells by rac activation. Biochem Biophys Res Commun 2000 269:247-253.

65. Churin Y, Kardalinou E, Meyer TF, Naumann M: Pathogenicity islanddependent activation of Rho GTPases Rac1 and Cdc42 in Helicobacter pylori infection. Mol Microbiol 2001, 40:815-823.

66. Brandt S, Shafikhani S, Balachandran P, Jin S, Hartig R, Konig W, Engel J, Backert S: Use of a novel coinfection system reveals a role for Rac1, HRas, and Crkll phosphorylation in Helicobacter pylori-induced host cell actin cytoskeletal rearrangements. FEMS Immunol Med Microbiol 2007, 50:190-205.

67. Tabassam FH, Graham DY, Yamaoka Y: OipA plays a role in Helicobacter pylori-induced focal adhesion kinase activation and cytoskeletal reorganization. Cell Microbiol 2008, 10:1008-1020.

68. Snider JL, Allison C, Bellaire BH, Ferrero RL, Cardelli JA: The beta1 integrin activates JNK independent of CagA, and JNK activation is required for Helicobacter pylori CagAt-induced motility of gastric cancer cells. J Biol Chem 2008, 283:13952-13963.

69. Schaller MD, Schaefer EM: Multiple stimuli induce tyrosine phosphorylation of the Crk-binding sites of paxillin. Biochem J 2001 360:57-66
70. Suzuki M, Mimuro H, Suzuki T, Park M, Yamamoto T, Sasakawa C: Interaction of CagA with Crk plays an important role in Helicobacter pylori-induced loss of gastric epithelial cell adhesion. J Exp Med 2005 202:1235-1247.

71. Muyskens JB, Guillemin K: Helicobacter pylori CagA disrupts epithelial patterning by activating myosin light chain. PLOS One 2011, 6:e17856.

72. Knauer O, Binai NA, Carra G, Beckhaus T, Hanschmann KM, Renne T, Backert S, Karas M, Wessler S: Differential phosphoproteome profiling reveals a functional role for VASP in Helicobacter pylori-induced cytoskeleton turnover in gastric epithelial cells. Cell Microbiol 2008, 10:2285-2296.

73. Selbach M, Moese S, Hauck CR, Meyer TF, Backert S: Src is the kinase of the Helicobacter pylori CagA protein in vitro and in vivo. J Biol Chem 2002, 277:6775-6778.

74. Stein M, Bagnoli F, Halenbeck R, Rappuoli R, Fantl WJ, Covacci A: c-Src/Lyn kinases activate Helicobacter pylori CagA through tyrosine phosphorylation of the EPIYA motifs. Mol Microbiol 2002, 43:971-980.

75. Hatakeyama M: Linking epithelial polarity and carcinogenesis by multitasking Helicobacter pylori virulence factor CagA. Oncogene 2008, 27:7047-7054.

76. Handa O, Naito Y, Yoshikawa T: CagA protein of Helicobacter pylori: a hijacker of gastric epithelial cell signaling. Biochem Pharmacol 2007, 73:1697-1702.

77. Moese S, Selbach M, Brinkmann V, Karlas A, Haimovich B, Backert S, Meyer TF: The Helicobacter pylori CagA protein disrupts matrix adhesion of gastric epithelial cells by dephosphorylation of vinculin. Cell Microbiol 2007, 9:1148-1161.

78. Higashi H, Tsutsumi R, Muto S, Sugiyama T, Azuma T, Asaka M, Hatakeyama M: SHP-2 tyrosine phosphatase as an intracellular target of Helicobacter pylori CagA protein. Science 2002, 295:683-686.

79. Higashi H, Nakaya A, Tsutsumi R, Yokoyama K, Fujii Y, Ishikawa S, Higuchi M, Takahashi A, Kurashima Y, Teishikata Y, Tanaka S, Azuma T, Hatakeyama M: Helicobacter pylori CagA induces Ras-independent morphogenetic response through SHP-2 recruitment and activation. J Biol Chem 2004 279:17205-17216

80. Yamazaki S, Yamakawa A, Ito Y, Ohtani M, Higashi H, Hatakeyama M, Azuma T: The CagA protein of Helicobacter pylori is translocated into epithelial cells and binds to SHP-2 in human gastric mucosa. J Infect Dis 2003, 187:334-337.

81. Tsutsumi R, Takahashi A, Azuma T, Higashi H, Hatakeyama M: Focal adhesion kinase is a substrate and downstream effector of SHP-2 complexed with Helicobacter pylori CagA. Mol Cell Biol 2006, 26:261-276.

82. Tegtmeyer N, Wittelsberger R, Hartig R, Wessler S, Martinez-Quiles N, Backert S: Serine Phosphorylation of Cortactin Controls Focal Adhesion Kinase Activity and Cell Scattering Induced by Helicobacter pylori. Cell Host Microbe 2011, 9:520-531.

83. Wessler S, Rapp UR, Wiedenmann B, Meyer TF, Schoneberg T, Hocker M Naumann M: B-Raf/Rap1 signaling, but not c-Raf-1/Ras, induces the histidine decarboxylase promoter in Helicobacter pylori infection. FASEB J 2002, 16:417-419.

84. Brandt S, Wessler S, Hartig R, Backert S: Helicobacter pylori activates protein kinase $C$ delta to control Raf in MAP kinase signalling: role in AGS epithelial cell scattering and elongation. Cell Motil Cytoskeleton 2009, 66:874-892.

85. Tammer I, Brandt S, Hartig R, Konig W, Backert S: Activation of Abl by Helicobacter pylori: a novel kinase for CagA and crucial mediator of host cell scattering. Gastroenterology 2007, 132:1309-1319.

86. Fu HY, Asahi K, Hayashi Y, Eguchi H, Murata H, Tsujii M, Tsuji S, Azuma T, Kawano S: East Asian-type Helicobacter pylori cytotoxin-associated gene A protein has a more significant effect on growth of rat gastric mucosal cells than the Western type. J Gastroenterol Hepatol 2007, 22:355-362.

\section{doi:10.1186/1478-811X-9-27}

Cite this article as: Wessler et al:: Regulation of the actin cytoskeleton in Helicobacter pylori-induced migration and invasive growth of gastric epithelial cells. Cell Communication and Signaling 2011 9:27. 